\title{
Real-Time Image Recognition and Path Tracking of a Wheeled Mobile Robot for Taking an Elevator
}

\author{
Jih-Gau Juang ${ }^{1}$, Chia-Lung Y ${ }^{2}$, Chih-Min Lin ${ }^{3}$, \\ Rong-Guan Yeh ${ }^{4}$, Imre J. Rudas ${ }^{5}$ \\ ${ }^{1,2}$ Department of Communications, Navigation and Control Engineering, National \\ Taiwan Ocean University, Keelung, 202, Taiwan, e-mail: jgjuang@ ntou.edu.tw \\ ${ }^{3,4}$ Department of Electrical Engineering, Yuan Ze University, Chung-Li, Taoyuan, \\ 320, Taiwan, e-mail: cml@saturn.yzu.edu.tw \\ ${ }^{5}$ Óbuda University, Bécsi út 96/B, H-1034 Budapest, Hungary, \\ e-mail: rudas@uni-obuda.hu
}

\begin{abstract}
This paper aims to design a wheeled mobile robot for path tracking and for automatically taking an elevator by integrating multiple technologies, including image processing using hue-saturation-value color space, pattern recognition using the adaptive resonance theory (ART), and robot control using a fuzzy cerebellar model articulation controller (FCMAC). The ART is used to recognize the button of the elevator. It is an effective competitive learning rule for figure identification. The FCMAC is used for path tracking. Experimental results demonstrate the proposed control system can drive the wheeled robot to take the elevator automatically.
\end{abstract}

Keywords: Pattern recognition; Adaptive resonance theory; Fuzzy CMAC; Wheeled mobile robot

\section{Introduction}

With the progress of human civilization, as well as lifestyle changes, intelligent robots are gaining influence in human daily life, such as providing entertainment, life safety, health and other aspects of the service. Intelligent robots integrate mechanics, electronics, automation, control, and communications technologies. Various types of robots have been developed in recent years. For a variety of needs, the development of a robot system combines many advanced theories, such as path planning, visual image processing technology, body positioning, obstacle avoidance techniques, and arm control. In recent years, the wheeled mobile robot 
(WMR) has been frequently discussed in mobile robot researches. It has some advantages, such as easy control, high-speed mobility, and energy storage capacity $[1,2]$, which are better than for the legged robot. WMR has been successfully applied to path planning, parking control, obstacle avoidance, navigation, object tracking, cooperation of multiple robots, etc.

Many researchers have tried to convert expertise into the robotic system so that the human-robot interaction in daily life can be more harmonious. Zhao and BeMent [3] addressed six common kinds of three-wheeled robot models and their ability of control. Leow et al. have analyzed the kinetic model of an all-direction wheeled robot [4]. Zhong used an omni-directional mobile robot on a map building application [5]. Chung et al. [6] utilized two wheels and different speeds for position control. Shi et al. installed multiple sensors on a WMR and utilized fuzzy reasoning value to control the WMR [7]. Path planning and fuzzy logic controller have been developed to make a WMR track a desired path [8]. In the development of the intelligent versatile automobile, the automobile used ultrasound sensors to help it search the correct reflective position in an unknown environment $[9,10]$.

The cerebellar model articulation controller (CMAC) can be thought of as a learning mechanism to imitate the human brain [11]. By applying the fuzzy membership function into a CMAC, a fuzzy CMAC has been created $[12,13]$. The advantages of a fuzzy CMAC over neural networks (NNs) have been presented [14, 15]. Thus, a fuzzy CMAC will be designed for the control scheme of a WMR.

This study aims to design an intelligent control strategy for a wheeled robot to track an assigned path and to take an elevator automatically so that it can be applied for the service of document delivery in a company. This study applies a fuzzy CMAC to a wheeled mobile robot to track a path and uses adaptive resonance theory [16] to identify an elevator button. Since the WMR is a nonlinear model, a dynamic equation is needed for system analysis and control regulation design. The fuzzy controller is one of the most prevalent controllers for nonlinear systems because the control scheme of a fuzzy controller is simple and flexible. A localization system called StarGazer [17] is used to provide coordinate locations for the WMR. For the fuzzy controller, its inputs are the coordinate and the heading angle. Therefore, the WMR can track a preset path accurately. A neural network system based on the adaptive resonance theory (ART) is utilized for pattern recognition. The neural network can identify the elevator button quickly and correctly. The contributions of this study are: (1) that an intelligent control scheme is proposed to control a WMR to take elevator automatically; (2) that the ART is utilized to recognize elevator buttons successfully; (3) that path planning is obtained by a localization system; and (4) that trajectory control is achieved by a fuzzy CMAC. 


\section{System Description}

The experiments are performed on the Ihomer WMR [18], as shown in Fig. 1.

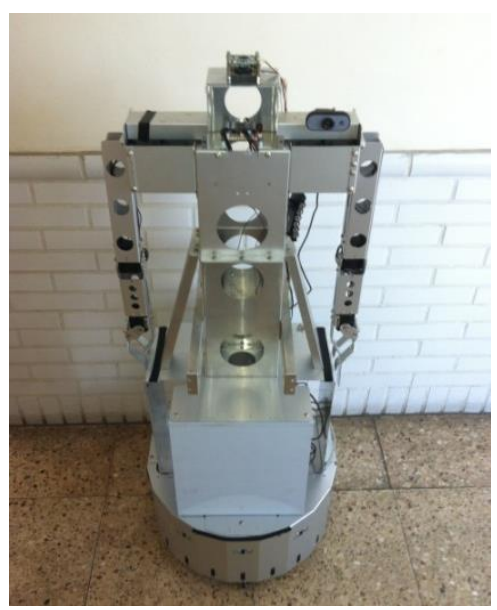

Figure 1

Ihomer wheeled mobile robot (WMR)

A StarGazer localization system is installed on top of the WMR to transmit and receive the infrared signals that are used to obtain the coordinate location and heading angle of the WMR. There are six ultrasonic sensors, of make Devantech SRF05, and the detecting range is from $1 \mathrm{~cm}$ to $4 \mathrm{~m}$. The SRF05 sonar sensor module is easy to connect with an ordinary control panel, such as BASICX, etc. The sonar frequency is $40 \mathrm{KHz}$, the current is $4 \mathrm{~mA}$, and the voltage is $5 \mathrm{~V}$. Assume the condition that the WMR is located on the Cartesian coordinate system (global coordinate system) as shown in Fig. 2, which has no lateral or sliding movement. The WMR represents a vector with nonlinear terms on the two dimensional Cartesian coordinate system carrying both head direction and lateral direction information. The WMR belongs to a nonholonomic system [19]. The movement change of WMR on a global coordinate is

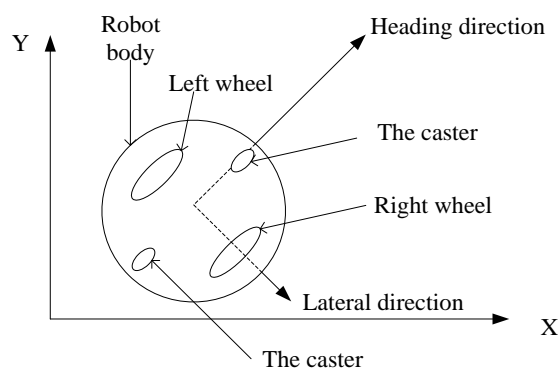

Figure 2

WMR coordinate diagram 


$$
\left(\begin{array}{c}
\dot{x} \\
\dot{y} \\
\dot{\theta}
\end{array}\right)=g_{1}(q) v+g_{2}(q) w=\left(\begin{array}{c}
\cos \theta \\
\sin \theta \\
0
\end{array}\right) v+\left(\begin{array}{l}
0 \\
0 \\
1
\end{array}\right) \omega
$$

If $\Delta t<<1$, (1) can be replaced by (2)

$$
\left[\begin{array}{l}
x_{k}(i+1) \\
y_{k}(i+1) \\
\theta(i+1)
\end{array}\right]=\left[\begin{array}{l}
x_{k}(i) \\
y_{k}(i) \\
\theta(i)
\end{array}\right]+\left[\begin{array}{cc}
\cos \theta & 0 \\
\sin \theta & 0 \\
0 & 1
\end{array}\right]\left[\begin{array}{l}
v \\
\omega
\end{array}\right] \Delta \mathrm{t}
$$

Definitions of all variables are given as follows:

$v$ : Speed of the center of the body

$w$ : Angular speed of the center of the body

$r$ : Radius of the wheel

$d$ : Distance between the left wheel and the right wheel

$x_{k}(i+1)$ : Next $\mathrm{x}$-axis coordinate of the center of the body on global coordinate system

$y_{k}(i+1):$ Next y-axis coordinate of the center of the body on global coordinate system

$x_{k}(i): \mathrm{X}$-axis coordinate of the center of the body on global coordinate system

$y_{k}(i)$ : Y-axis coordinate of the center of the body on global coordinate system

$\Delta t$ : Sample time of every movement of the WMR

$w_{l}$ : Left-wheel speed of the WMR

$w_{r}$ : Right-wheel speed of the WMR

In order to obtain the relative distance, we change the target position and the WMR position from the global coordinate system to the local coordinate as shown in Fig. 3, which is between the center of the WMR and the expected position.

$$
\left[\begin{array}{c}
x_{t}^{\prime} \\
y_{t}^{\prime}
\end{array}\right]=\left[\begin{array}{cc}
\sin \theta & -\cos \theta \\
\cos \theta & \sin \theta
\end{array}\right]\left[\begin{array}{l}
x_{t}-x_{k} \\
y_{t}-y_{k}
\end{array}\right]
$$

The WMR center relative distance and expected position (error distance) is

$$
d_{e}=\sqrt{x_{t}^{2}+y_{t}^{\prime 2}}
$$




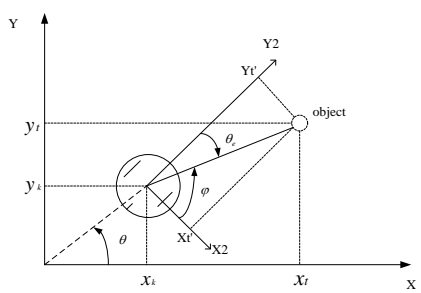

Figure 3

Coordinate of relative position of the WMR and expected position

The relative angle is defined as the included angle between the heading direction of the WMR and the expected position. The local coordinates of the WMR obtained by (5) through the trigonometric function can be calculated.

$$
\varphi=\tan ^{-1}\left(\frac{y_{t}^{\prime}}{x_{t}^{\prime}}\right)
$$

The error angle of the heading direction and expected position is calculated by (6).

$$
\theta_{e}=90-\varphi
$$

Definitions of all variables are given as follows:

$X$ : $\mathrm{X}$-axis of the global coordinate system

$Y$ : $\mathrm{Y}$-axis of the global coordinate system

$X 2$ : $\mathrm{X}$-axis of the body coordinate system

Y2: Y-axis of the body coordinate system

$X t$ : The $\mathrm{x}$-axis coordinate of the expected position on the global coordinate system

$Y t$ : The $y$-axis coordinate of the expected position on the global coordinate system

$X \mathrm{k}$ : The $\mathrm{x}$-axis coordinate of the center of the body on the global coordinate system

$Y \mathrm{k}$ : The $\mathrm{y}$-axis coordinate of the center of the body on the global coordinate system

$X t^{\prime}$ : The $\mathrm{x}$-axis coordinate of the expected position on the body coordinate system

$Y t^{\prime}$ : The $y$-axis coordinate of the expected position on the body coordinate system

$\theta$ : The included angle between the center of the body and the x-axis of the global coordinate system

$\theta e$ : The relative angle of the heading direction of the WMR and the expected position (error angle)

$\varphi$ : The included angle between the lateral direction of the WMR and the expected position

$d e$ : The relative distance between the WMR and the expected position (error distance) 


\section{Image Process}

The HSV color space is used. The color is made up of its hue, saturation, and brightness. Hue describes where the color is found in the color spectrum and the shade of the color. Red, yellow and purple are used to describe the hue. The saturation describes how pure the hue is with respect to a white reference. The HSV color of external light can improve the identifiable rate of color and the speed of image process [20].

The RGB image of the elevator buttons is shown in Fig. 4(a). The RGB color space values are transferred to the HSV color space values [21] with the following equations:

$$
\begin{aligned}
& H=\cos ^{-1} \frac{(R-G)+(R-B)}{\sqrt{(R-B)^{2}+(R-B)}(G-B)}, B \leq G \\
& H=2 \pi-\cos ^{-1} \frac{(R-G)+(R-B)}{\sqrt{(R-B)^{2}+(R-B)}(G-B)}, B>G \\
& S=\frac{\max (R, G, B)-\min (R, G, B)}{\max (R+G+B)} \quad, \quad V=\frac{\max (R, G, B)}{255}
\end{aligned}
$$

$\mathrm{V}$ plane is used first. Because the figure that needs identifying remains largely unchanged, a threshold is set to the image and then this image can be processed to become a binary case. Then, open and closed image filtering is used to make the image clearer. This is a very common way of filtering. The processed image is shown in Fig. 4(b).

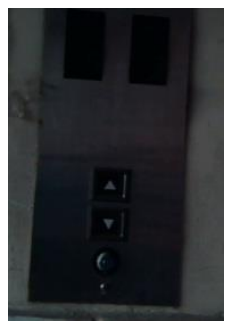

(a)

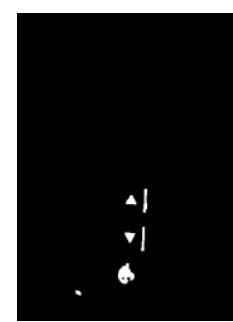

(b)

Figure 4

The elevator buttons and the processed images

Next, the target image will be identified by the ART method. The ART neural network is shown in Fig. 5. 


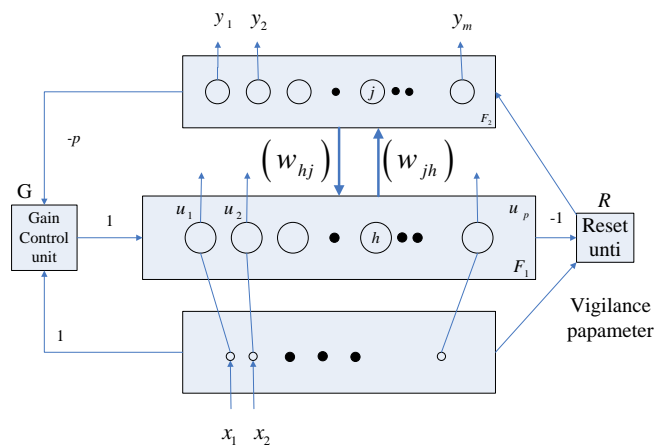

Figure 5

ART neural network

Procedures of the ART neural network are shown as follows:

Step 1: Set all neurons with the initial bounded values.

Step 2: Present the input pattern to the network. Note that the first image pattern is set to the first neuron as the winner and is assigned to class 1 , and then skip to Step 6.

Step 3: Enable the neurons that have won and have been assigned to certain classes.

Step 4: According to the following criteria, to find the closest class with the input vector, the so-called "closest" refers to the "similarity". The similarity measurement is defined as (the first rating standard):

$$
S_{1}\left(\underline{w}_{j}, \underline{x}\right)=\frac{\underline{w}_{j} \cdot \underline{x}}{\beta+\left\|\underline{w}_{j}\right\|_{1}}=\frac{\underline{w}_{j}^{T} \underline{x}}{\beta+\sum_{i=1}^{p} w_{i j}}
$$

Step 5: From Step 4, select the winner that has the largest $S_{1}$. Assume the $j^{\text {th }}$ neuron is the winner, then the second similarity standard is applied to measure the victorious neuron samples stored in the neurons. The second similarity measurement is defined as the evaluation standard (the second rating standard):

$$
S_{2}\left(\underline{w}_{j}, \underline{x}\right)=\frac{\underline{w}_{j} \cdot \underline{x}}{\|\underline{x}\|_{1}}=\frac{\underline{w}_{j}^{T} \underline{x}}{\sum_{i=1}^{p} x_{i}}
$$

When $S_{2}\left(\underline{w}_{j}, \underline{x}\right) \geq \rho$ ( $\rho$ is appositive constant and is less than 1 , and is called vigilance parameter), the $w_{j}$ and $x$ can be regard as very similar, then we can perform Step 6; otherwise the $j^{\text {th }}$ neuron is disabled, go back to Step 4, find the next winning neuron. 
Step 6: Adjust the weights of the winner neuron $j$ by following equation:

$$
\underline{w}_{j}(n+1)=\underline{w}_{j}(n) \text { AND } \underline{x} \sqrt{b^{2}-4 a c}
$$

The output of neuron $j$ represents the input pattern $\underline{x}$ is recognized as class $j$. Go back to Step 2 to re-accept next new input pattern.

\section{Control Scheme}

In this study, the control scheme is divided into two parts. The first part is path tracking and the other part is a process to control the robot to push and check the elevator button. A fuzzy CMAC is developed to realize the path tracking control. A linear controller is used to control the WMR in the push-and-check button mission, and another linear controller is used to control the arm's position by the target point with image processing. The control sequence is shown in Fig. 6.

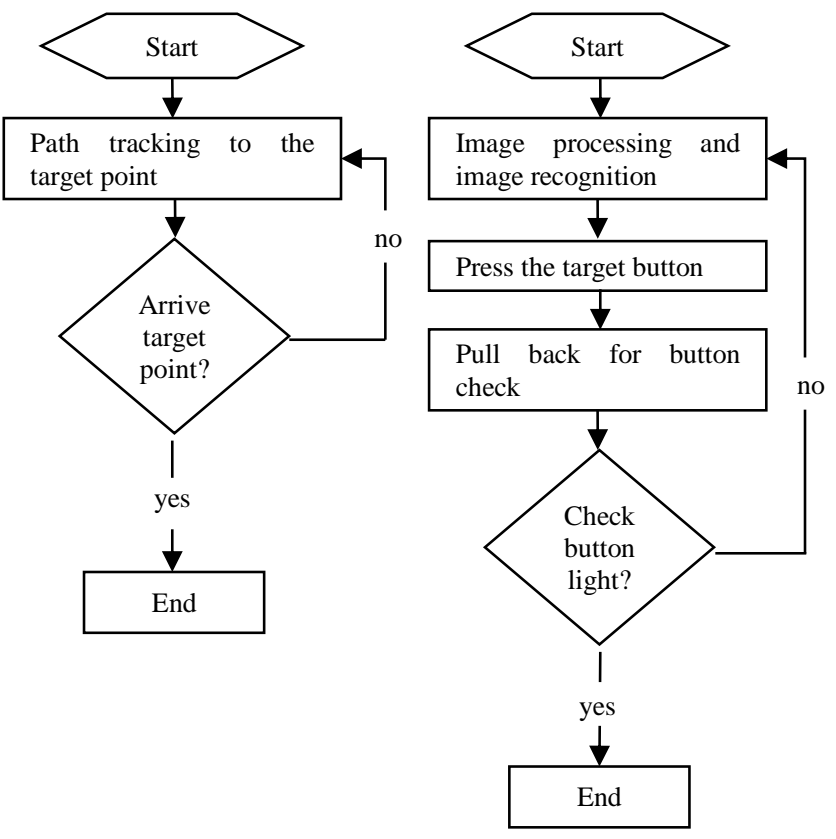

Figure 6

Flowchart of the control sequence 


\section{A Path Planning}

In this study, a localization system is applied in path planning. In order to make the path clear, we need to paste landmarks on the ceiling every 150 centimeters and even in the elevator. With the coordinates obtained by the localization system, we can plan the desired path for the WMR, as shown in Fig. 7.

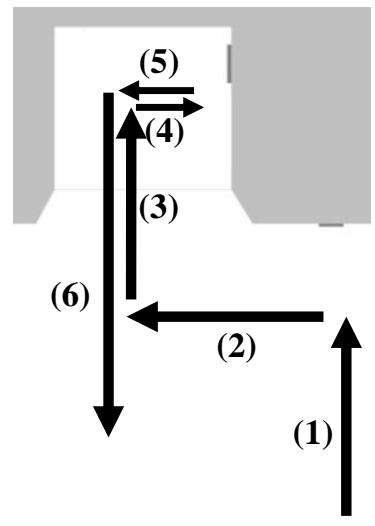

Figure 7

The planning paths

\section{B Fuzzy CMAC Control}

In order to take the elevator automatically, the WMR uses the fuzzy CMAC to track the desired path and uses image processing and image recognition to find the elevator button. The considered FCMAC is shown in Fig. 8. Compared with a neural network, the FCMAC has a faster learning rate and better local generalization ability. The processing algorithms of the FCMAC are described as follows:

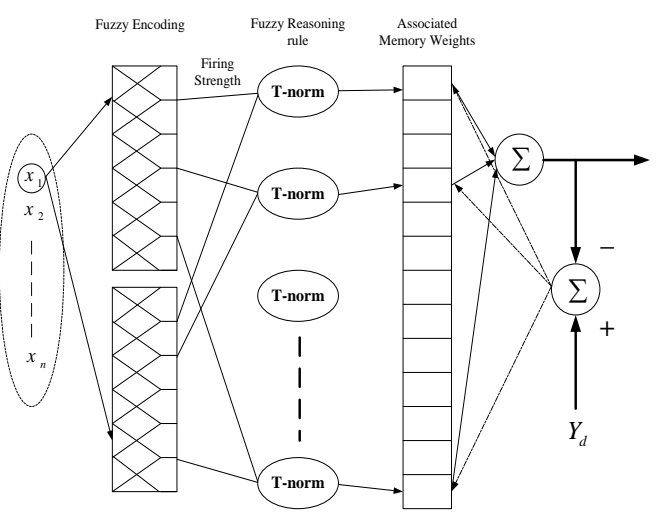

Figure 8

Conventional FCMAC structure 


\section{Step 1:}

Quantization $(X \rightarrow S): X$ is an n-dimension input space. For the given, $x=\left[\begin{array}{ll}x_{1} & x_{2}\end{array}\right.$ $\left., \ldots, x_{n}\right]^{T}, s=\left[s_{1} s_{2}, \ldots, s_{n}\right]^{T}$ represents the quantization vector of $x$. It is specified as the corresponding state of each input variable before the fuzzification.

Step 2:

Associative Mapping Segment $(S \rightarrow C)$ : It is to fuzzify the quantization vector which is quantized from $x$. After the input vector is fuzzified, the input state values are transformed to "firing strength" based on the corresponding membership functions.

Step 3:

Memory Weight Mapping $(C \rightarrow W)$ : The $i^{\text {th }}$ rule's firing strength in the FCMAC could be computed as:

$$
C_{j}(x)=c_{j 1}\left(x_{1}\right) * c_{j 2}\left(x_{2}\right) * . . c_{j n}\left(x_{n}\right)=\prod_{i=1}^{n} c_{j 1}\left(x_{i}\right)
$$

where $c_{j_{i}}\left(x_{i}\right)$ is the $j^{\text {th }}$ membership function of the $i^{\text {th }}$ input vector and $n$ is the number of total states. The asterisk "*” denotes a fuzzy $t$-norm operator. Here the product inference method is used as the $t$-norm operator.

Step 4:

Output Generation with Memory Weight Learning $(W \rightarrow Y)$ : Due to partial proportional fuzzy rules and existent overlap situation, more than one fuzzy rule is fired simultaneously. The consequences of the multi-rules are merged by a defuzzification process. The defuzzification approach we applied is to sum assigned weights of the activated fuzzy rules on their firing strengths, denoted as $C_{j}(x)$. The output of the network is,

$$
y=\sum_{j=1}^{N}\left(w_{j} C_{j}(x) / \sum_{i=1}^{N} C_{i}(x)\right)
$$

The weight update rule for FCMAC is as follows:

$$
w_{j}^{(i)}=w_{j}^{(i-1)}+\frac{\alpha}{m}\left(y_{d}-y\right) C_{j}(x) / \sum_{i=1}^{N} C_{i}(x)
$$

where $\alpha$ is the learning rate, $m$ is the size of floor (called generalization), and $y_{d}$ is the desired output. Here, an adaptive learning rate is introduced [22]. Let the tracking error $e(t)$ be

$$
e(t)=y_{d}-y(t)
$$


where $t$ is the time index. A Lyapunov function can be expressed as

$$
V=\frac{1}{2} e^{2}(t)
$$

Thus, the change in the Lyapunov function is obtained by

$$
\Delta V=V(t+1)-V(t)=\frac{1}{2}\left[e^{2}(t+1)-e^{2}(t)\right]
$$

The error difference can be represented by

$$
\Delta e(t) \approx\left[\frac{\partial e(t)}{\partial W}\right] \cdot \Delta W(t)
$$

Using the gradient descent method, we have

$$
\Delta w_{j}(t)=-\frac{\alpha}{m} \frac{\partial V(t)}{\partial w_{j}(t)}
$$

Since

$$
\frac{\partial V(t)}{\partial w_{j}(t)}=e(t) \cdot \frac{\partial e(t)}{\partial w_{j}(t)}=\left(y_{d}-y(t)\right) \cdot\left(-C(x)^{T} / \Sigma C(x)\right)
$$

Thus

$$
\begin{aligned}
\Delta w_{j}(t) & =\frac{\alpha}{m}\left(C_{j}(x) / \Sigma C(x)\left(y_{d}-y(t)\right), j=1,2, \ldots \ldots, N\right. \\
\Delta W(t) & =\left[\begin{array}{c}
\Delta w_{i}(t) \\
\Delta w_{2}(t) \\
\vdots \\
\Delta w_{N}(t)
\end{array}\right]=\frac{\alpha}{m}\left[\begin{array}{c}
\left(C_{1}(x) / \Sigma C(x)\right. \\
\left(C_{2}(x) / \Sigma C(x)\right. \\
\vdots \\
\left(C_{N}(x) / \Sigma C(x)\right.
\end{array}\right] \cdot\left(y_{d}-y(t)\right) \\
& =\frac{\alpha}{m} \cdot\left(C(x) / \Sigma C(x) \cdot\left(y_{d}-y(t)\right)\right.
\end{aligned}
$$

From (13) and (15) we have

$$
\frac{\partial e(t)}{\partial w_{j}(t)}=-C_{j}(x) / \Sigma C(x), \frac{\partial e(t)}{\partial W}=-C(x)^{T} / \Sigma C(x)
$$


From (16) to (23) we have

$$
\begin{aligned}
& \Delta V=\frac{1}{2}\left[e^{2}(t+1)-e^{2}(t)\right] \\
& =\frac{1}{2}[e(t+1)-e(t)] \cdot[e(t+1)+e(t)] \\
& =\frac{1}{2}[\Delta e(t)] \cdot[2 \cdot e(t)+\Delta e(t)] \\
& =\Delta e(t) \cdot\left[e(t)+\frac{1}{2} \Delta e(t)\right] \\
& =\left[\frac{\partial e(t)}{\partial W} \cdot \frac{\alpha}{m} \cdot(C(x) / \Sigma C(x)) \cdot e(t)\right] \cdot \\
& \left\{e(t)+\frac{1}{2}\left[\frac{\partial e(t)}{\partial W} \cdot \frac{\alpha}{m} \cdot(C(x) / \Sigma C(x)) \cdot e(t)\right]\right\}
\end{aligned}
$$

Since $\frac{\partial e(t)}{\partial W}=-C(x)^{T} / \Sigma C(x)$, we have

$$
\begin{aligned}
& \Delta V=\left[-C(x)^{T} / \Sigma C(x) \cdot \frac{\alpha}{m} \cdot C(x) / \Sigma C(x) \cdot e(t)\right] \\
& \left\{e(t)+\frac{1}{2}\left[-C(x)^{T} / \Sigma C(x) \cdot \frac{\alpha}{m} \cdot C(x) / \Sigma C(x) \cdot e(t)\right]\right\} \\
& =\left[-\frac{1}{2} \frac{\alpha}{m} \cdot e(t) \cdot \frac{C(x)^{T} \cdot C(x)}{(\Sigma C(x))^{2}}\right] \cdot\left[2 e(t)-\frac{\alpha}{m} \cdot e(t) \cdot \frac{\left\|C(x)^{2}\right\|}{(\Sigma C(x))^{2}}\right] \\
& =\left[-\frac{1}{2} \frac{\alpha}{m} \cdot e^{2}(t) \cdot \frac{\left\|C(x)^{2}\right\|}{(\Sigma C(x))^{2}}\right] \cdot\left[2-\frac{\alpha}{m} \cdot \frac{\left\|C(x)^{2}\right\|}{(\Sigma C(x))^{2}}\right]
\end{aligned}
$$

Let $\left[2-\frac{\alpha}{m} \cdot \frac{\left\|C(x)^{2}\right\|}{(\Sigma C(x))^{2}}\right]>0$ then $\Delta V<0$, i.e., select the learning rate as

$$
\frac{2 \cdot m \cdot(\Sigma C(x))^{2}}{\left\|C(x)^{2}\right\|}>\alpha>0
$$

This implies that $e(t) \rightarrow 0$ for $t \rightarrow \infty$. Thus, the convergence of the adaptive FCMAC learning system is guaranteed. 


\section{Experiment Results}

First, the path to the elevator is planned with an indoor localization system. The WMR moves along the path and reaches the desired location in front of the elevator buttons and then uses the webcam to obtain button images. After using image recognition to find location information, the WMR moves its robot arm to reach the target altitude and presses the desired button. The WMR then pulls back and checks the button light. With the indoor localization system the WMR moves along the planned route to reach the elevator door and uses ultrasonic sensors to detect the elevator door. After the elevator door opens, the WMR moves into the elevator and tracks the preset path so that it can reach the desired location in front of elevator buttons. Path tracking is shown in Fig. 9.

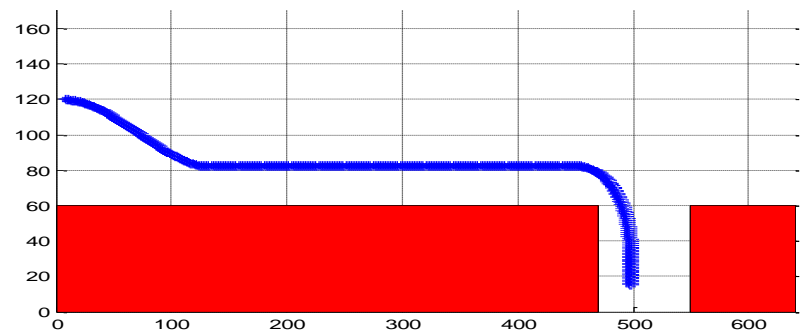

Figure 9

WMR path tracking by FCMAC

With image identification, the WMR moves its robot arm and presses the desired button, and then it waits for the elevator door to open. When the elevator door opens, the WMR moves out from the elevator, and then all actions are completed.

When taking the elevator, the WMR starts at the outside of the elevator. First, the WMR gets its own coordinates via the StarGazer. The WMR moves along the desired path to the front of the outside button of the elevator by path tracking until the WMR moves to the stop line. In the second part, the WMR raises its left arm and points to the target button. When the WMR pushes the outside button, it checks the button lights and moves backward to the stop line, as shown in Fig. 10.

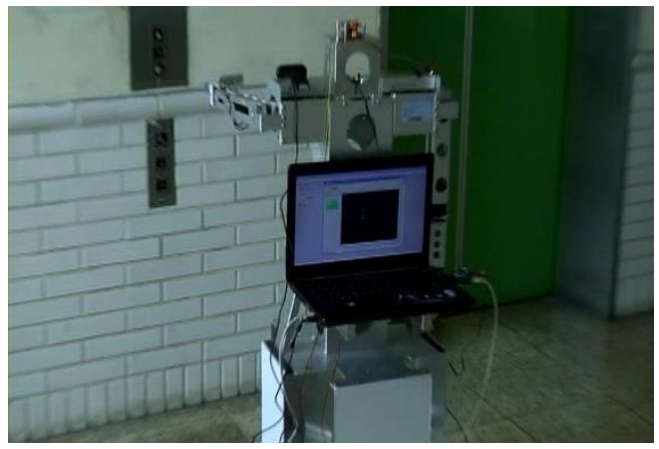



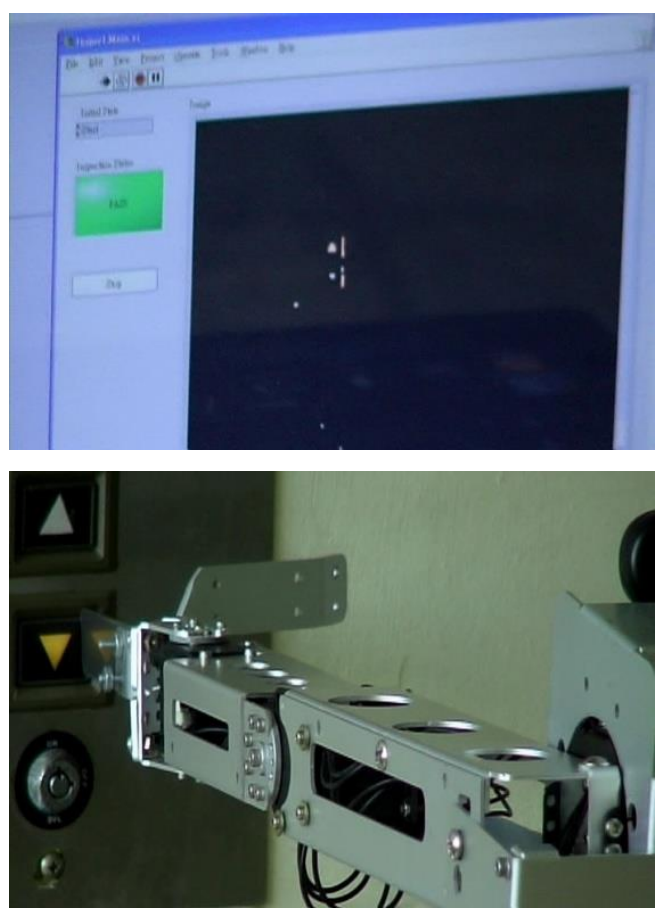

Figure 10

Push the outside button and identify it

In the third part, the WMR moves to the position in front of the elevator. This mission needs to use the coordinates from the StarGazer. With the coordinates, the WMR can move close to the desire path, as shown in Fig. 11.

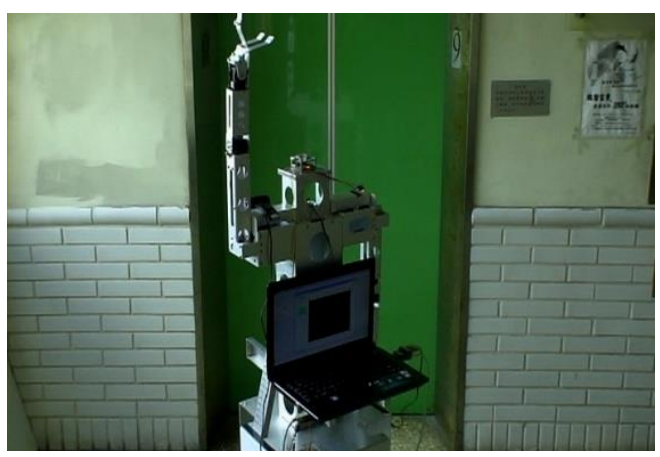




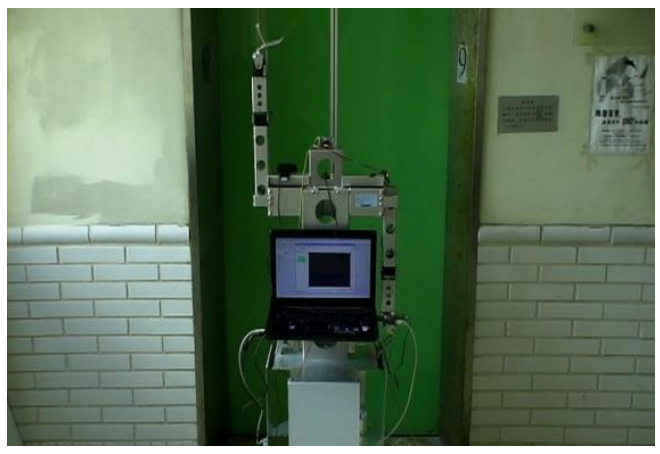

Figure 11

Move to the position in front of the elevator

In the fourth part, the WMR waits in front of the door, and when the door opens the WMR moves to the target position, which is in front of the elevator button, as shown in Fig. 12.
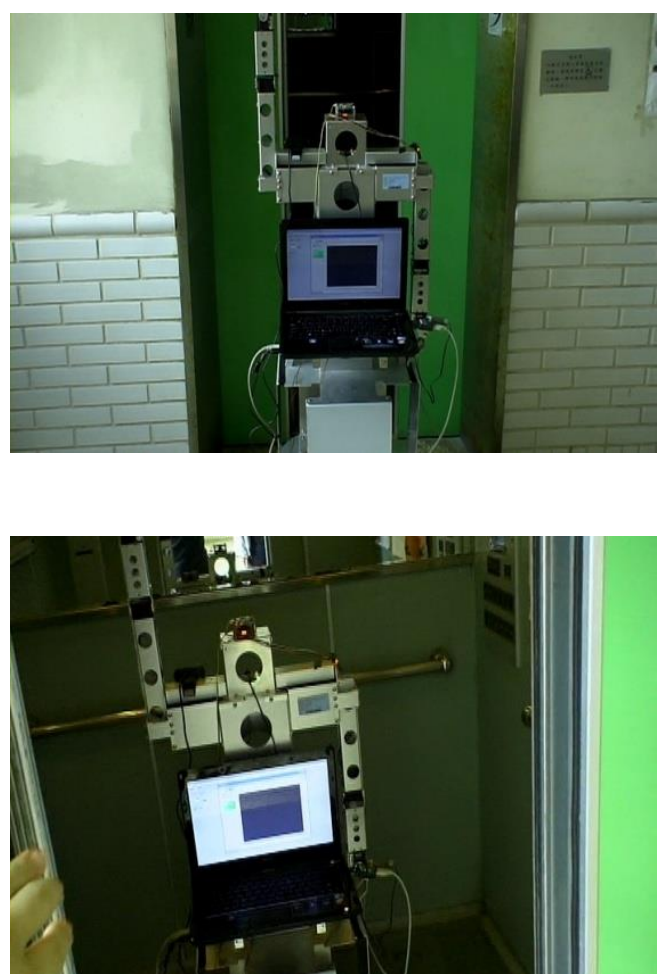

Figure 12

Wait for the door open and move into the elevator 
In the fifth part, the WMR pushes the inside button of the elevator and checks the button light. First, the WMR raises its left arm and points to the target button. Second, when the WMR pushes the inside button, it identifies the button lights and move backward to the stop line, as shown in Fig. 13.
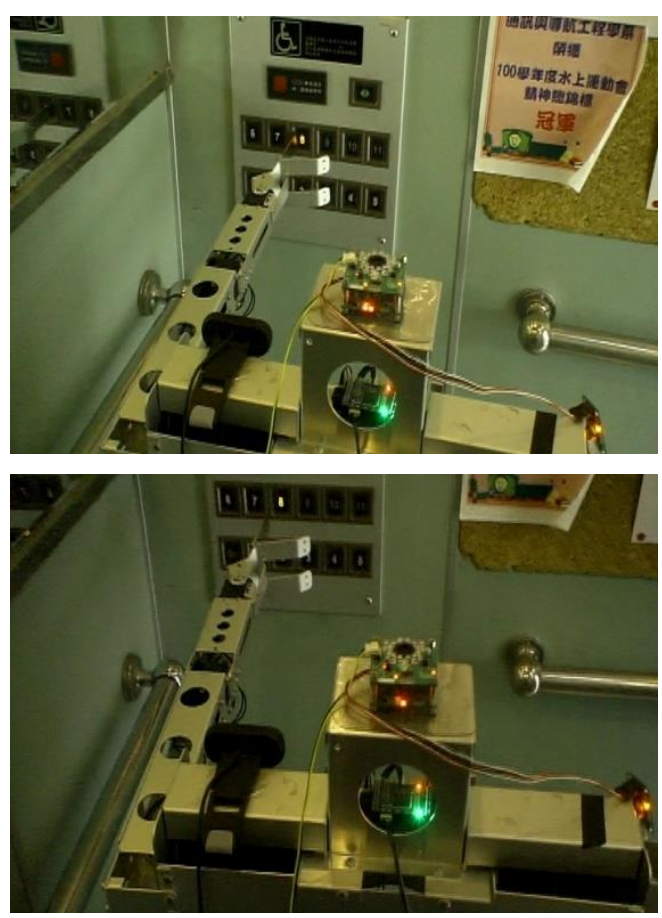

Figure 13

Push the inside elevator button and identify it

In the sixth part, the WMR moves to the position in front of the elevator door, waits for the door to open and moves out, as shown in Fig. 14. Then the complete processes are finished.

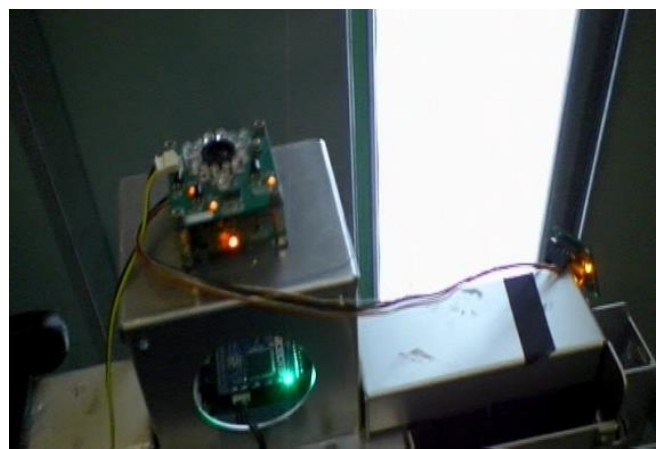




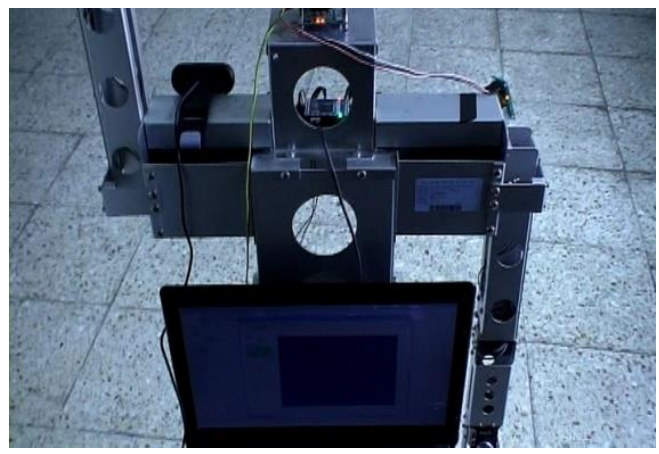

Figure 14

Move out of the elevator

\section{Conclusions}

In this paper, an intelligent control scheme based on FCMAC control, image processing, and adaptive resonance theory is proposed to control a mobile robot for path tracking and automatically taking an elevator. This research integrates a localization system, StarGazer, and ultrasonic sensors to track the desired path and distinguish whether the elevator door is opened or not. With the coordinate provided by the StarGazer and the controller, the WMR can move along the desired path. In image processing, the interference of light intensity is very troublesome. Therefore, the HSV color space is used to solve interference of light. The webcam vibrates while the WMR moves and this will cause the captured image to be unstable and with noise. This problem can be solved with filtering processes. Experiment results show the developed control system can drive the wheeled robot to track the desired path and to take the elevator successfully.

\section{References}

[1] J. J. Zhan, C. H. Wu, J. G. Juang: Application of Image Process and Distance Computation to WMR Obstacle Avoidance and Parking Control, $20105^{\text {th }}$ IEEE Conference on Industrial Electronics and Applications, pp. 1264-1269, 2010

[2] A. Rodic, G. Mester: Sensor-based Navigation and Integrated Control of Ambient Intelligent Wheeled Robots with Tire-Ground Interaction Uncertainties, Journal of Applied Science, Acta Polytechnica Hungarica, Vol. 10, No. 3, pp. 114-133, 2013

[3] Y. Zhao and S. L. BeMent: Kinematics, Dynamics and Control of Wheeled Mobile Robots, 1992 IEEE International Conference on Robotics \& Automation, 1992, pp. 91-96

[4] Y. P. Leow, K. H. Low, and W. K. Loh: Kinematic Modelling and Analysis of Mobile Robots with Omni-Directional Wheels, The Seventh International Conference on Control, Automation, Robotics and Vision, 2002, pp. 820825 
[5] Q. H. Zhong: Using Omni-Directional Mobile Robot on Map Building Application, Master Thesis, Department of Engineering Science, NCKU, ROC, 2009

[6] Y. Chung and C. Park, and F. Harashima: A Position Control Differential Drive Wheeled Mobile Robot, IEEE Transactions on Industrial Electronics, Vol. 48, No. 4, pp. 853-863, 2001

[7] E. X. Shi, W. M. Huang, and Y. Z. Ling: Fuzzy Predictive Control of Wheeled Mobile Robot Based on Multi-Sensors, The Third International Conference on Machine Learning and Cybernetics, 2004, pp. 439-443

[8] T. H. Lee, H. K. Lam, F. H. F. Leung, and P. K. S. Tam: A Practical Fuzzy Logic Controller for The Path Tracking of Wheeled Mobile Robots, IEEE Control Systems Magazine, 2003, pp. 60-65

[9] P. Bai, H. Qiao, A. Wan, and Y. Liu: Person-Tracking with Occlusion Using Appearance Filters, The 2006 IEEE/RSJ International Conference on Intelligent Robots and Systems Beijing, China, October 9-15, 2006, pp. 1805-1810

[10] T. Darrell, G. Gordon, M. Harville, J. Woodfill: Integrated Person Tracking Using Stereo, Color, and Pattern Detection, Conference on Computer Vision and Pattern Recognition, 1998, pp. 601-609

[11] J. S. Albus: A New Approach to Manipulator Control: The Cerebellar Model Articulation Controller (CMAC)," J. Dynamic System, Measurement and Control, Vol. 97, No. 3, pp. 220-227, Sep. 1975

[12] C. T. Chiang, and C. S. Lin: CMAC with General Basis Functions," Neural Netw., Vol. 9, No. 7, pp. 1199-1211, 1996

[13] Y. F. Peng and C. M. Lin: Intelligent Motion Control of Linear Ultrasonic Motor with $\mathrm{H}^{\infty}$ Tracking Performance," IET Contr. Theory and Appl., Vol. 1, No. 1, pp. 9-17, Jan. 2007

[14] P. E. M. Almedia, and M. G. Simoes: Parametric CMAC Network Fundamentals and Applications of a Fast Convergence Neural Structure," IEEE Trans. Industrial Application, Vol. 39, No. 5, pp. 1551-1557, 2003

[15] C. M. Lin, L. Y. Chen, and C. H. Chen: RCMAC Hybrid Control for MIMO Uncertain Nonlinear Systems Using Sliding-Mode Technology," IEEE Trans. Neural Network, Vol. 18, No. 3, pp. 708-720, May 2007

[16] G. A. Capenter and S. Grossberg: Adaptive Resonance Theory, CAS/CNS Technical Report, 2009-008, 2009

[17] User's Guide, Localization system StarGazer ${ }^{\mathrm{TM}}$ for Intelligent Robots. HAGISONIC Co., LTD, 2004

[18] idRobot iHomer USER MANUA, http://www.idminer.com.tw 
[19] B. Dandrea, G. Bastin, and G. Campion: Dynamic Feedback Linearization of Nonholonomic Wheeled Mobile Robots, The 1992 IEEE International Conference on Robotics \& Automation, 1992, pp. 820-825

[20] C. Bunks, The RGB Color space, http://gimp-savvy.com/ BOOK/index. html?node50.html

[21] C. Bunks: The HSV Color space, http://gimpsavvy.com/BOOK/index.html/node50.html

[22] C. M. Lin and H. Y. Li: A Novel Adaptive Wavelet Fuzzy Cerebellar Model Articulation Control System Design for Voice Coil Motors, IEEE Transactions on Industrial Electronics, Vol. 59, 2012, No. 4, pp. 2024-2033 